\title{
Development steps of 2-color laser-induced fluorescence with MDR-enhanced energy transfer for instantaneous planar temperature measurement of micro-droplets and sprays
}

\author{
Johannes Palmer*, Manuel A. Reddemann, Valeri Kirsch, Reinhold Kneer \\ ${ }^{1}$ Institute of Heat and Mass Transfer, RWTH Aachen University, Germany \\ *Corresponding author: palmer@wsa.rwth-aachen.de
}

\begin{abstract}
A new method for instantaneous measurement of temperature, size and velocity of micro-droplets has been developed. The method is based on the well-known 2-color laser-induced fluorescence (2cLIF) technique, but uses a pulsed laser for 2-dimensional imaging without motion blur and an adjusted dye mixture for suppression of LIFMDRs by utilizing the MDR-enhanced energy transfer effect. This work presents the development steps that are necessary to verify feasibility of pulsed $2 D$-2cLIF-EET for micro droplet and hollow-cone spray applications.
\end{abstract}

\section{Keywords}

droplets, temperature, 2cLIF, morphology dependent resonances, Pyrromethene 597-8C9, Oil Blue N

\section{Introduction}

Temperature measurements of micro droplets are an important tool to understand various physical processes, e.g. mixture formation of fuel and air in an engine's cylinder. Most contact-free temperature measurement approaches for micro droplets are based on the so called 2-color laser-induced fluorescence (2cLIF) thermometry method developed at LEMTA [1]. The technique uses a fluorescence dye dissolved in a liquid of interest. After excitation by a continuous wave $(\mathrm{CW})$ laser, the dye re-emits absorbed laser light as spontaneous fluorescence with temperature dependent intensity ${ }^{1}$ (Fig 1, A). Fluorescence intensity is evaluated in two wavelength bands (two colors): one wavelength band with a strong and one with weak temperature dependence. The ratio of both signals remains temperature sensitive, but is ideally independent of local and temporal fluctuations in laser light excitation, droplet size and dye concentration.[1] Fluorescence from two separate wavelength bands may be recorded by different types of detector-configurations [2]:

- OD-2cLIF uses two photomultiplier tubes (PMT) for point-wise fluorescence detection (first shown by [1]).

- 1D-2cLIF is based on a spectrograph coupled to an intensified CCD. The detector's entrance slit allows one dimensional fluorescence detection. Fluorescence bands can be flexibly selected during post-processing [2, 3]. As a CW laser is used for excitation, recorded images are affected by motion blur.

- 2D-2CLIF uses two CCDs for two dimensional (planar) fluorescence detection. Motion blur becomes a major issue due to long CCD exposure duration and processes can only be investigated on average: e.g. images of a micro droplet chain appear jet-like shaped with the width of the droplets [4] or in case of spray investigations, turbulent effects such as vortices are lost [5].

To explore more specific types of problems, basic 2cLIF has been continuously extended or modified: e.g. different types of fluorophores for various liquids $[6,7]$ or a third PMT for an additional wavelength band that accounts for self-absorption effects of a single dye [8]. More interestingly, latest 2cLIF publications consider a pulsed laser for fluorescence excitation [2, 9, 10]. Pulsed lasers solve the issue of motion blur. In case of a planar detectorconfiguration, instantaneous measurements of temperature and size are possible. However, high excitation power of a pulsed laser inevitably leads to so called morphology dependent resonances (MDRs) inside micro droplets $[3,11]$. MDRs occur regardless of the presence of any fluorescent dye. If a fluorescent dye is present, its emission spectrum is changed by the MDRs and reliable 2cLIF thermometry is prevented [2, 12, 13]. The term MDR describes light being trapped inside a droplet due to almost total internal reflection at the droplet's inner surface and going into resonances. The droplet acts as micro-cavity and causes constructive interference of light waves with an increased photon density of state [11]. A higher density of state increases the probability of nonlinear optical processes. In fact, fluorescence starts to resonate during MDR occurrence as well. This phenomenon is called MDR-lasing (or simply lasing) and is caused by the increased density of state. The dye's cross-section becomes increased and stimulated fluorescence emission starts as soon as the cavity round-trip gain succeeds cavity losses due to leakage and absorption [14]. This is always the case for high excitation power provided by a pulsed laser. The process of lasing is represented in images by ring-like bright structures at the droplet surface with two additional opposing bright spots in case of plane wave illumination (Fig. 1, C). The bright spots are a consequence of the super-position of light circling in standing waves around the droplet surface.[14] Modes of light resonances become visible at specific frequencies in the dye's emission spectrum (Fig 1, B), depending on droplet size [15], physical properties [16] and excitation power $[15,17]$.

${ }^{1}$ 2cLIF-theory is specifically formulated for spontaneous fluorescence emission of a single dye only. 
A spontaneous emission of droplets $\lambda_{11} \lambda_{12} \lambda_{21} \lambda_{22}$

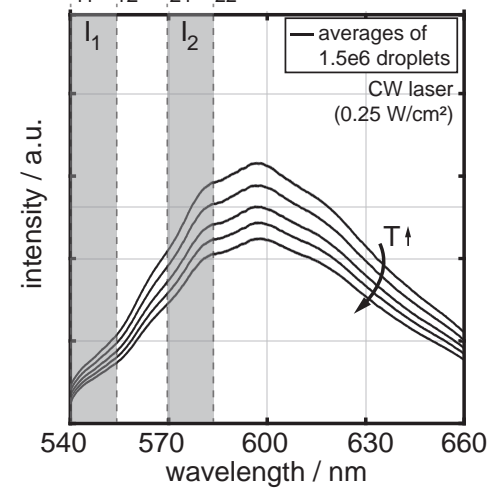

B MDRs-lasing of droplets

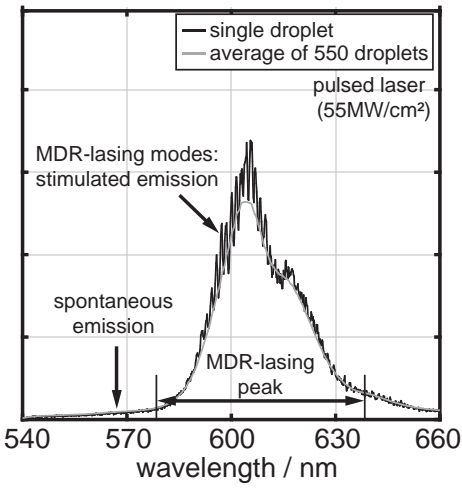

C droplet image with MDRs

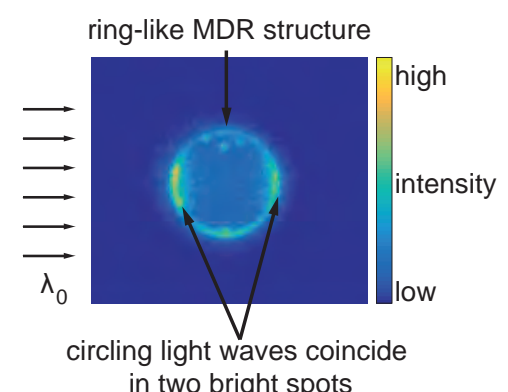

in two bright spots

Figure 1. Fluorescence spectra and images of $70 \mu \mathrm{m}$ droplets consisting of PM597 dissolved in Ethanol (10 $\mu \mathrm{M})$. Details about the measurement setup are listed at a later section. Diagram A: Illumination by a CW laser causes spontaneous emission. Wavelength regions $\left(I_{1}, I_{2}\right)$ for 2cLIF thermometry are highlighted. Diagram B: Pulsed excitation causes MDRs. Fluorescence spectra become a super-position of spontaneous and stimulated emission. A single droplet spectrum allows identification of MDR modes. Image C: MDR-lasing appears as ring-like structure with two additional bright spots.

MDR-lasing makes 2cLIF thermometry unfeasible, especially for planar detection, where bright intensity peaks limit the usability of intensified CCDs. In order to clear the emission spectrum of pyrromethene 597-8C9 (PM597) from MDR-lasing, Perrin et al. suggest using an additional dye called Oil Blue N (OBN) [13]. The two-dye combination allows MDR-enhanced energy transfer (EET) from one dye to another. Energy that would originally be spend on PM597-lasing is transferred to OBN instead. In turn, lasing occurs for OBN at higher wavelengths. Spectroscopic pulsed 1D-2cLIF measurements approve this theory and also show that pulsed 2cLIF thermometry is feasible.[2]. Until now, pulsed 2D-2cLIF with MDR-enhanced energy transfer has not been tested on droplets or sprays. However, due to the importance of temperature determination of sprays, more simple 2cLIF setups have been tested: Occurrence of MDRs has only been considered in a single publication [12], where liquid spray temperature is determined point-wise using CW-OD-thermometry. The same type of setup has been used in numerous approaches afterwards, but without consideration of MDR-lasing (e.g. [18]). The average temperature field of a hollow cone spray has been measured by Mishra et al. [5] using CW-2D-2cLIF in combination with SLIPI ${ }^{2}$. MDR-lasing has not been discussed in that work. Only a single pulsed 2D-2cLIF approach has been found in commonly available literature [9]. The pulsed laser that is used in the approach definitively causes MDR-lasing of the fluorophor, but the phenomenon has not been considered at all.

In the following, basic 2cLIF theory is explained and experimental setups are described. Afterwards, three development steps are presented, describing how pulsed 2D-2cLIF with MDR-enhanced energy transfer (pulsed 2D2cLIF-EET) is designed: i) Dyes need to be selected for targeted MDR-enhanced energy transfer and tested in well defined droplet chain experiments using 1D-spectroscopy. ii) The 2cLIF setup is upgraded with a planar detectorconfiguration (pulsed 2D-2cLIF-EET). The setup is tested in well-defined droplet experiments. iii) The new setup is put to the test in with a complex hollow cone-spray under engine-like conditions.

\section{Theory of 2CLIF thermometry for spontaneous emission of a single dye}

$2 \mathrm{cLIF}$ thermometry requires a fluorescent dye with wavelength dependent temperature sensitivity. Fig. 1(A) shows fluorescence emission of pyrromethene 597-8C9 for a set of temperatures measured in bulk. The dye's spontaneous emission spectrum $I(\lambda)\left[\mathrm{W} / \mathrm{m}^{2}\right]$ can be described by eq. 1 . The equation contains an Arrhenius approach to model temperature dependence of the absorption cross-section and quenching effects [1]:

$$
I(\lambda)=K_{\mathrm{opt}}(\lambda) K_{\mathrm{spec}}(\lambda) V I_{0} C \exp \left[\frac{\beta(\lambda)}{T}\right]
$$

The equation contains optical and spectral constants $K_{\mathrm{opt}}(\lambda)$ [-] and $K_{\mathrm{spec}}(\lambda)$ [1/mol], the illuminated measurement volume in the focal plane $V\left[\mathrm{~m}^{3}\right]$, excitation intensity $I_{0}\left[\mathrm{~W} / \mathrm{m}^{2}\right]$, temperature $T[\mathrm{~K}]$ and the dye's molecular concentration $C\left[\mathrm{~mol} / \mathrm{m}^{3}\right.$ ] and temperature sensitivity $\beta(\lambda)[\mathrm{K}][6]$. For a wavelength range from $\lambda_{i 1}$ to $\lambda_{i 2}$, fluorescence $I_{i}$ is described by the integral $[1,6]$

$$
I_{i}=\int_{\lambda_{i 1}}^{\lambda_{i 2}} K_{\mathrm{opt}}(\lambda) K_{\mathrm{spec}}(\lambda) V I_{0} C \exp \left[\frac{\beta(\lambda)}{T}\right] \mathrm{d} \lambda=K_{\mathrm{opt}, i} K_{\mathrm{spec}, i} V I_{0} C \exp \left[\frac{a_{i}}{T^{2}}+\frac{b_{i}}{T}\right]
$$

where $\beta(\lambda)$ is approximated by a second order polynomial with coefficients $a_{i}$ and $b_{i}$. The 2cLIF thermometry approach uses the ratio $R(T)$ [-] of two different wavelength regions $\left(I_{1}, I_{2}\right)$, to cancel out fluctuating values such as excitation intensity $I_{0}$, measurement volume $V$ and dye concentration $C$ [1]:

$$
R(T)=\frac{I_{2}}{I_{1}}=\frac{K_{\mathrm{opt}, 2} K_{\text {spec }, 2}}{K_{\mathrm{opt}, 1} K_{\mathrm{spec}, 1}} \exp \left[\frac{a_{2}-a_{1}}{T^{2}}+\frac{b_{2}-b_{1}}{T}\right]=\exp \left[\frac{a_{21}}{T^{2}}+\frac{b_{21}}{T}+c\right]
$$

\footnotetext{
${ }^{2}$ Structured Laser Illumination Planar Imaging
} 
This simplified equation contains only three unknown parameters: temperature sensitivities $a_{21}\left[\mathrm{~K}^{2}\right], b_{21}[\mathrm{~K}]$ and setup constant $c[-]$. All parameters are determined by calibration at known temperatures. Wavelength bands are selected by evaluating the measured temperature sensitivity $\beta(\lambda)$ of a dye (see [6, 2] for details). One wavelength band is selected as temperature independent reference $\left(I_{1}\right.$ in Fig. $\left.1(\mathrm{~A})\right)$, the other wavelength band must be strongly temperature dependent $\left(I_{2}\right)$.

The theory above is derived for spontaneous fluorescence emission of a single dye only. This boundary condition is not necessarily given for pulsed laser excitation of dye-seeded micro droplets due to occurrence of MDR-lasing. Nevertheless, our latest publication has proven validity of this theory for pulsed excitation combined with a two dye MDR-enhanced energy transfer [2]. Therefore, validity of 2cLIF theory is assumed to be given for this works pulsed $2 D-2 c L I F-E E T$ setup as well.

\section{Experimental setups}

Four slightly different experimental setups are used for the pulsed planar 2cLIF development: i) \& ii) CW \& pulsed $1 \mathrm{D}$ spectroscopy of droplets iii) pulsed 2D-2cLIF of droplets and iv) pulsed 2D-2cLIF of hollow-cone sprays.
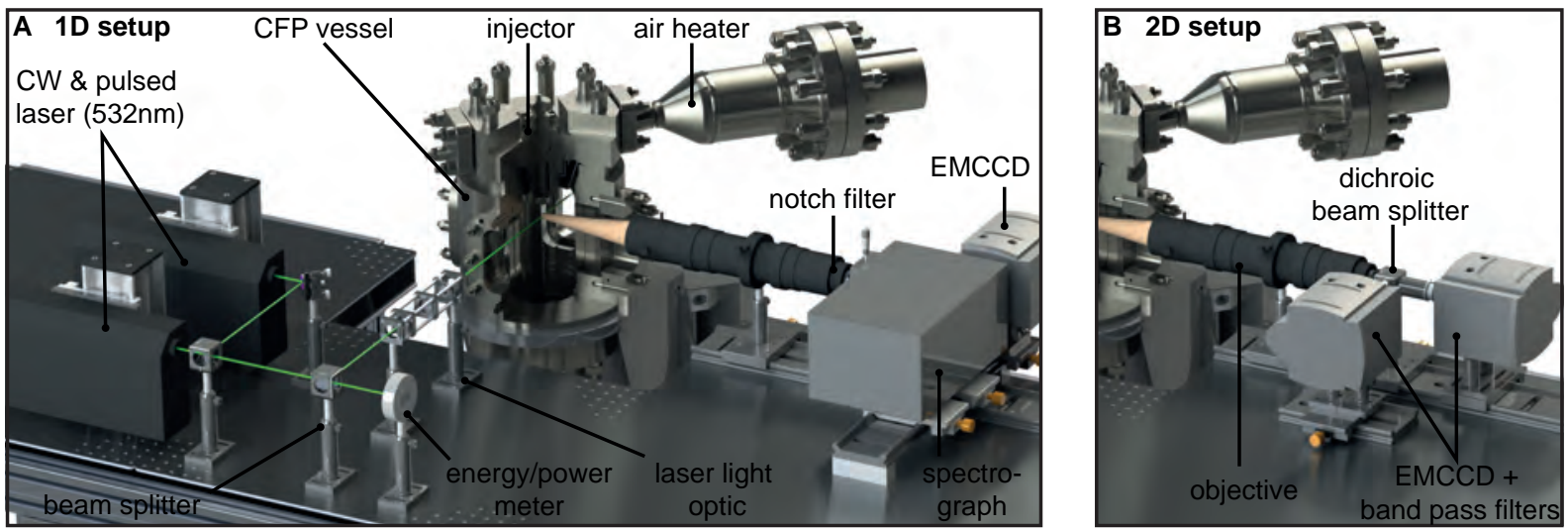

Figure 2. Experimental setup contains either a pulsed or CW laser for fluorescence excitation. Before the beam reaches the droplets, its cross section is expanded to about the size of the detector. After passing a high pass filter, a fluorescence image is magnified with a far-field microscope. The detector is a spectrograph that is connected to an electron multiplied CCD.

Micro droplets are produced by a vibrating orifice generator with an outlet diameter of $35 \mu \mathrm{m}$. Droplets with a size of approximately $70 \mu \mathrm{m}$ consist of Ethanol and are created with a constant frequency of $77 \mathrm{kHz}$. Droplets are injected into quiescent air with a pressure of 2.5 bar, corresponding to a measured droplet velocity of about $10.5 \mathrm{~m} / \mathrm{s}$. Liquid temperature is controlled by a heating cartridge with an integrated thermocouple. Cooling is provided by a surrounding water circuit. The droplet generator is placed in the central position of the head of a heatable constant pressure-flow (CPF) vessel (Fig. 2, A). The CPF chamber is only required for spray experiments. Ambient conditions remain atmospheric during the droplet experiments (293 K, 1 bar). The droplet measurement position lies $6 \mathrm{~mm}$ under the generator's outlet. This proximity guarantees isothermal conditions of liquid inside the injector and droplets. During spray experiments, a piezo-driven hollow-cone injector replaces the droplet generator. Ethanol as fuel is injected with a pressure of 150 bar and an injection duration of $2 \mathrm{~ms}$. The injector is temperature controlled at $305 \mathrm{~K}$ by a water circuit. The true injector outlet temperature is assumed to be higher due to heated air flowing $(<0.1 \mathrm{~m} / \mathrm{s})$ through the chamber. Experiments are conducted with an ambient temperatures of $400 \mathrm{~K}$ and an ambient pressure of 10 bar.

Table 1. Optical setting of the experimental setups.

\begin{tabular}{c|cccc}
\hline & CW 1D-spec. & pulsed 1D-spec. & pulsed 2D-2cLIF & pulsed 2D-2cLIF \\
droplets & droplets & droplets & spray \\
\hline illumination spot & circular beam & circular beam & circular beam & light sheet \\
illumination dimension & $\varnothing 7 \mathrm{~mm}$ & $\varnothing 2.7 \mathrm{~mm}$ & $\varnothing 2.7 \mathrm{~mm}$ & $0.3 \mathrm{~mm} \times 50 \mathrm{~mm}$ \\
$I_{0}$ & $0.25 \mathrm{~W} / \mathrm{cm}^{2}$ & $55 \mathrm{MW} / \mathrm{cm}^{2}$ & $55 \mathrm{MW} / \mathrm{cm}^{2}$ & $55 \mathrm{MW} / \mathrm{cm}^{2}$ \\
exposure time & $1 \mathrm{~s}$ & $10 \mu \mathrm{s}$ & $10 \mu \mathrm{s}$ & $10 \mu \mathrm{s}$ \\
image height & $2.15 \mathrm{~mm}$ & $2.15 \mathrm{~mm}$ & $2.15 \mathrm{~mm}$ & $47.89 \mathrm{~mm}$ \\
image width & - & - & $2.15 \mathrm{~mm}$ & $47.89 \mathrm{~mm}$ \\
magnification & 6.2 & 6.2 & 6.2 & 0.61 \\
image resolution & $0.477 \mathrm{px} / \mu \mathrm{m}$ & $0.477 \mathrm{px} / \mu \mathrm{m}$ & $0.477 \mathrm{px} / \mu \mathrm{m}$ & $0.0467 \mathrm{px} / \mu \mathrm{m}$ \\
wavelength scale & $7.24 \mathrm{px} / \mathrm{nm}$ & $7.24 \mathrm{px} / \mathrm{nm}$ & - & - \\
images per condition & 20 & 50 & 200 & 30 (injections) \\
\hline
\end{tabular}

A continuous wave (CW) and a pulsed Nd:YAG laser (pulse duration: $4 \mathrm{~ns}$ ) are available for fluorescence excitation, 
both operating at a wavelength of $532 \mathrm{~nm}$ and identical polarization (Fig. 2, A). The CW laser is applied for spectroscopic reference experiments of micro droplet chains only, where spontaneous fluorescence emission must be guaranteed. The pulsed laser is operated differently for micro droplet chain and spray investigations. Micro droplets are excited with a laser beam (flat-top intensity profile) and a pulse repetition rate of $2.5 \mathrm{~Hz}$. The laser beam is reshaped to a light sheet for the spray experiments and synchronized to the injection events. The light sheet allows to illuminate a thin cut-out section of the spray's hollow-cone. The specific laser excitation intensity of the pulsed laser is kept constant during droplet chain and spray experiments. Changes of the specific excitation intensity due to a different illumination spot size (circular beam for droplet and light sheet for spray) are compensated by alteration of the laser output energy. Laser output power is monitored continuously using a beam splitter that separates $15 \%$ of the laser output intensity (laser stability deviation $<3 \%$ ). See table 1 for details of the laser configuration.

Fluorescence of micro droplets is collected by a far-field microscope (Infinity optics K2/SC) (Fig. 2, A). A Notch filter (Thorlabs NF533-17) removes scattered laser light from the spectrum. The spectroscopic setup that is used for droplet investigations only, uses a spectrograph (Princeton Instruments, Acton SP 2300i) attached to an electron multiplied CCD (EMCCD, Andor iXon3 DU-888) for fluorescence evaluation. The spectrograph's entrance slit is opened far enough to collect light from entire droplets $(1 \mathrm{~mm})^{3}$. The 2D-2cLIF imaging setup uses two identical EMCCDs (model as before) for fluorescence detection (Fig. 2, B). After passing the Notch filter, fluoresce is separated at $573 \mathrm{~nm}$ wavelength using a dichroic beam splitter (Chroma ZT561rdc). Each light path is further band pass filtered before reaching the EMCCDs (Semrock 549/15 nm BL, Semrock, 586/15 nm BL). Band pass filters (wavelength bands) are selected in accordance with our previous investigations of PM597 [2]. EMCCDs amplify detected light with a gain of $200 \mathrm{x}$ and are cooled to $178 \mathrm{~K}$ for an optimum signal to noise ratio. Spray investigations require a larger field of view and thus a switching of the microscope for a macro objective (Zeiss Makro-Planar T 100mm ZF.2). Depending on the particular setup, EMCCDs record a different number of images to justify reproducibility. Image numbers and details of the optical setup are listed in table 1.

\section{Development steps of pulsed 2D-2cLIF with MDR-enhanced energy transfer}

Step I: Dye selection and 1D-spectroscopic verification of MDR-enhanced energy transfer for droplet chains Pyrromethene 597-8C9 (PM597) is selected as temperature sensitive fluorescent dye for all experiments. The dye is dissolved in Ethanol with a molar concentration of $10 \mu \mathrm{M}^{4}$. PM597 is known for its low probability of self-absorption [6], sufficient solubility and an invariable emission spectrum for many liquids [7]. Fig. 1(A) illustrates the emission behavior of the dye for spontaneous emission at different temperatures and the spectrum change due to MDR-lasing (B). In order to clear the temperature dependent wavelength region of PM597 from lasing, MDR-enhanced energy transfer is enabled by adding Oil Blue $\mathrm{N}(\mathrm{OBN})$ with a concentration of also $10 \mu \mathrm{M}$ [2].

A single dye absorbance (cuvette)

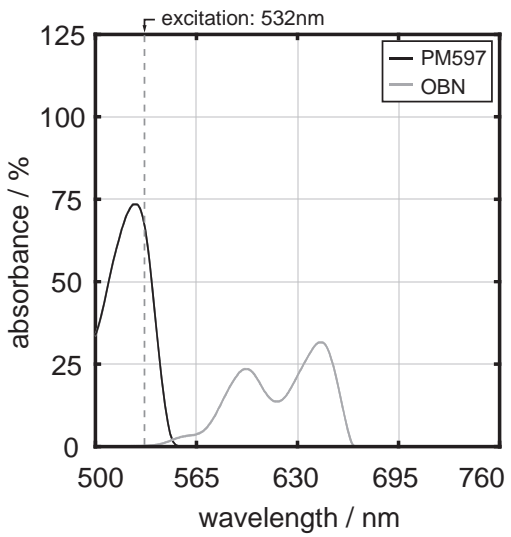

B single dye emission (cuvette)

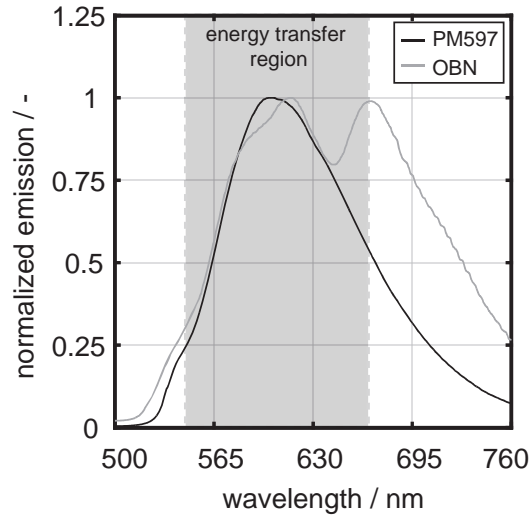

C average droplet chain emission

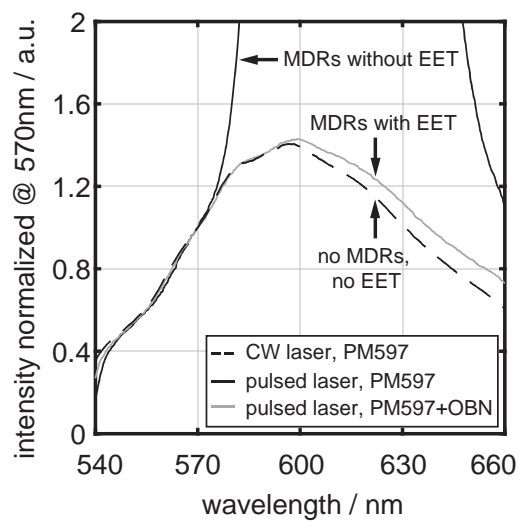

Figure 3. In bulk absorbance (A) and emission (B) spectra of pyrromethene 597-8C9 and Oil Blue N dissolved in Ethanol (each $10 \mu \mathrm{M}$. Diagram C: droplet chain fluorescence emission of an PM597-OBN mixture with and without MDR-EET in comparison with an MDR-free PM597 reference spectrum (operating conditions are listed in the previous chapter). Droplet results are averaged.

OBN is hardly excited by laser light (Fig. 3, A), such that energy absorption occurs almost exclusively by energy transfer from PM597 [2]. However, the shown emission spectrum of OBN (Fig. 3, B) is based on an excitation wavelength of $532 \mathrm{~nm}^{5}$. An energy transfer of both dyes takes place in the overlap region of the emission spectrum of PM597 and the absorption spectrum of $\mathrm{OBN}^{6}$ (compare Fig. $3 \mathrm{~A} \& \mathrm{~B}$ ). The major part of transferred energy is subsequently quenched or self-absorbed and only a minor part of energy is re-emitted. OBN emission based on energy transfer produces a much more red-shifted spectrum compared to the graph shown in Fig. 3(B), because dye excitation is not monochromatic and occurs at wavelengths much higher than $532 \mathrm{~nm}$. An energy transfer occurring

\footnotetext{
${ }^{3}$ The relatively large slit-width causes spectral averaging that needs to be remembered during result interpretation.

${ }^{4}$ The unit $\mu \mathrm{M}$ is a common abbreviation for $\mu \mathrm{mol} / \mathrm{L}$.

${ }^{5}$ Showing normalized emission removes the fact that absolute emission of OBN is almost negligible compared to PM597.

${ }^{6}$ Energy transfer happens only by radiation not by Förster resonant energy transfer, since molecule distances are large (low concentrations).
} 
in a droplet would cause an emission of OBN that includes a high amount of energy originally responsible for PM597lasing (compare Fig. 1, B), but becomes transferred to OBN before visible emission. As a consequence, PM597lasing is suppressed and OBN starts lasing instead, whereas lasing wavelengths are significantly red-shifted. One important condition for complete suppression of PM597-lasing is a targeted matching of both dye concentrations [2]. The selected concentrations of $10 \mu \mathrm{M}$ per dye, or rather their ratio of one, secure similar absorption crosssections and thus comparable probabilities of absorption. Stimulated emission of PM597 becomes interrupted and no cavity-round trip is completed.

Fig. 3(C) shows a comparison of fluorescence emission of droplets with and without energy transfer ${ }^{7}$. The emission spectrum with MDR-EET (pulsed laser, PM597+OBN) proves complete suppression of PM597-lasing. Due to the energy transfer, emission is a super-position of both dyes. Up to wavelengths of $600 \mathrm{~nm}$, the normalized emission spectrum is identical to spontaneous emission of PM597 that is based on CW excitation. Negligible OBN emission is caused by three facts: i) comparably weak emission of PM597, ii) weak absorption of OBN and iii) a strong self-absorption tendency of OBN. For wavelengths higher than $600 \mathrm{~nm}$, energy transfer conditions are different. Emission of OBN becomes visible in addition to PM597's fluorescence. Lasing that occurs for OBN now, is not visible in the given diagram, but definitively takes place for wavelengths higher than $660 \mathrm{~nm}^{8}$.

Validity of 2cLIF theory for the chosen dye concentrations and subsequent energy transfer has been proven in our previous investigations [2]: although EET causes saturation of spontaneous fluorescence emission in the 2cLIF wavelength bands, self-similarity of the normalized emission spectrum remains and thus 2cLIF theory yields. This conclusion is valid regardless of the chosen excitation intensity and droplet size. [2]

\section{Step II: Applicability of pulsed 2D-2cLIF with MDR-enhanced energy transfer for micro droplets}

Spectroscopic investigations of the chosen dye combination (PM597 and OBN, both seeded with $10 \mu \mathrm{M}$ in Ethanol) proof successful shifting of MDR-lasing from PM597 to OBN and validity of 2cLIF theory. In this development step, the spectrograph is replaced by two EMCCDs to enable imaging of temperature fields (Fig. 2, B). Stereo camera vision requires calibration to secure an identical field of view. Images of a reference target are matched using bi-linear interpolation. Calibration results are applied to every set of recorded images. The calibration is highly sensitive to miss-alignments, such that mistakes on the sub-pixel scale result in severe measurement errors (see [4] for details). After successful camera calibration, images of both devices are divided pixel by pixel. Camera calibration is followed by a temperature calibration that requires measurements of average droplet intensity ratios for a set of known temperatures under isothermal conditions. Average droplet ratios are determined as the follows: i) Droplets of each ratio-image are isolated and saved as images one after another in an array, whereas droplet centers are aligned. ii) The array is averaged to receive a locally resolved average droplet ratio-image. A following occurrence-refining ${ }^{9}$ improves the image quality at the droplet surface. iii) Further horizontal and vertical image averaging allows determining an overall droplet-ratio for each temperature (Fig. 4, B).

A average images of isothermal droplets

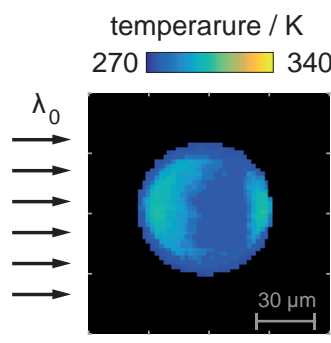

temperature: $285 \mathrm{~K}$

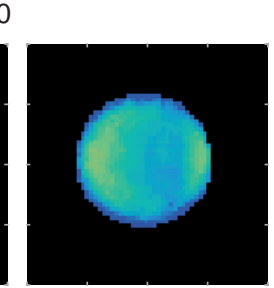

$297 \mathrm{~K}$

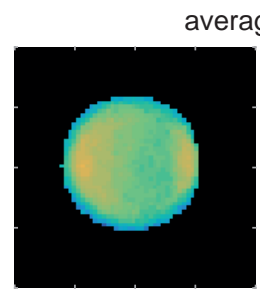

$309 \mathrm{~K}$ averages of 2700 droplets

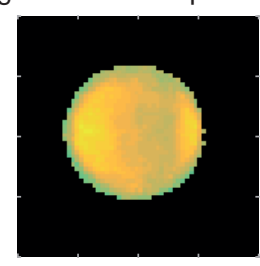

$321 \mathrm{~K}$
B overall temperature calibration

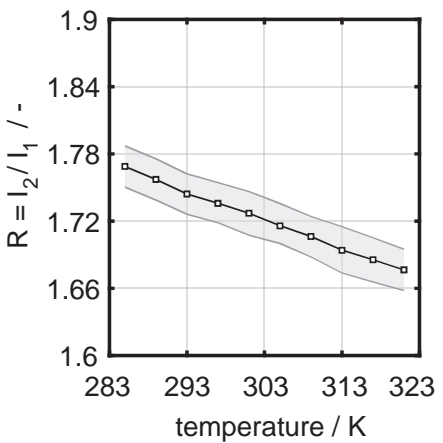

Figure 4. Temperature calibration results of the pulsed 2D-2cLIF-EET droplet investigation. Images (A): Average droplet temperature images under isothermal condition are affected by a nonphysical, but systematic temperature fluctuation across the droplet. The cause of this effect is still unknown. Diagram (B): The corresponding temperature calibration that is used to convert ratio into temperature images. Uses experimental operating conditions are listed in the section Experimerntal setups.

The measured temperature-ratio function is used to determine polynomial constants in eq. 3 . Eq. 3 is then applied pixel-wise to ratio-images to convert them into temperature fields. Fig. 4(A) shows average temperature-images of isothermal droplets. Although different temperatures can clearly be distinguished, an unexpected inhomogeneity pattern is evident for each measured temperature. The inhomogeneity explains the standard deviation of the calibration that corresponds to about 3 to $4 \mathrm{~K}$. Locally increased temperatures are found to be nonphysical, because the laser pulse itself causes only a negligible temperature change of $\Delta T=0.07 \mathrm{~K}$ for the given experimental configura$\operatorname{tion}^{10}$. The cause of the emerged inhomogeneity pattern is more likely caused by a locally changed laser excitation intensity that affects the fluorescence spectrum of one detection wavelength band stronger than the other. Excitation

\footnotetext{
${ }^{7}$ How to evaluate CW and pulsed 1D-spectroscopic images can be found at [2].

${ }^{8}$ This fact will be shown in a future work.

${ }^{9}$ Averaged pixel values that are based on information of less than half of the total number of evaluated droplets are sorted out.

${ }^{10}$ This value can be determined by a simple approximation.
} 
intensity may be reduced due to strong light absorption by the dyes on the light entering side of the droplet (concentration effect). On the opposing side, laser intensity may be increased due to focusing effects as the droplet acts as lens (hypothesis according to [19]). Nevertheless, the cause for changes in the fluorescence emission spectrum due to fluctuations in laser intensity remains unknown and requires further research.

In total, pulsed 2D-2cLIF-EET of micro droplets shows convincing results. Although droplet temperature-images show evidence of an unexplained physical mechanism, average droplet temperature can be reliably measured. Applying pulsed 2D-2cLIF-EET for a macroscopic case, i.e. a spray, makes influences of inhomogeneities inside droplets negligible. Reduced optical magnification makes droplets appear as objects smaller than a single pixel with a single average intensity value. Moreover, the amount of droplets and the depth of field contribute to a further averaging. Applying pulsed 2D-2cLIF-EET for a complex spray seems therefore feasible.

\section{Step III: Applicability of pulsed 2D-2cLIF with MDR-enhanced energy transfer for hollow-cone sprays}

In the third development step, pulsed 2D-2cLIF-EET is applied to a complex macroscopic droplet phenomenon: a poly-disperse, evaporating hollow-cone spray. Dye seeded Ethanol is injected into heated and pressurized air (400 K, 10 bar). EMCCDs record single-shot images for four different moments of the injection (from $0.5 \mathrm{~ms}$ to $2.0 \mathrm{~ms}$ in steps of $0.5 \mathrm{~ms}$ ). For each of the four moments, 30 consecutive fuel injections are recorded with a time separation of five seconds. After stereo-camera calibration, temperature calibration is conducted by placing a closed cuvette filled with fuel in the focal measurement plane. Step-wise increased ambient air temperature heats up the liquid to isothermal and known temperatures (up to the normal boiling point of Ethanol, $351 \mathrm{~K}$ ). Measurements lead to a linear calibration (similar to Fig. 4, B), but with low standard deviation. An additional calibration is performed for varying ambient pressure, showing no intensity-ratio change for the tested range up to 15 bar.

A

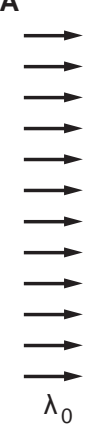

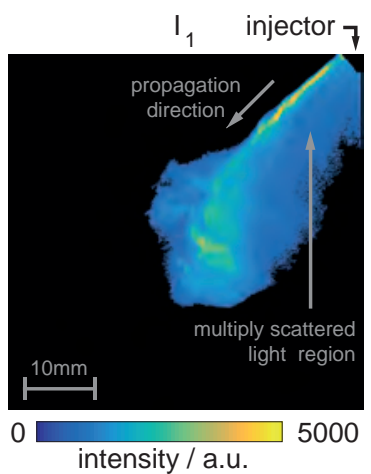

intensity / a.u.

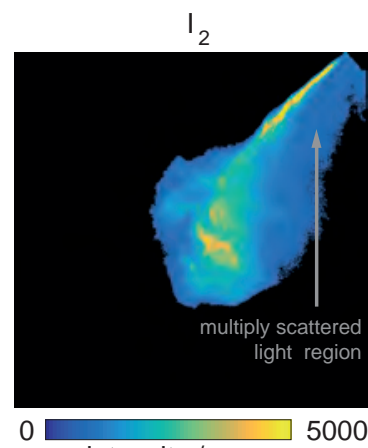

intensity / a.u.
B

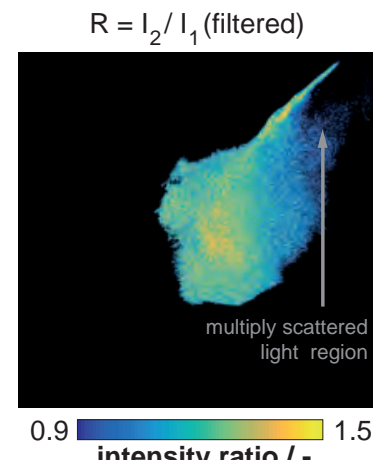

Figure 5. A shows both single-shot intensity images of the hollow-cone spray 2 ms after start of injector energizing. Dense spray regions are identified by high intensity values. Disruptive multiply scattered light causes severe "noise". B shows the corresponding single-shot ratio image. Ratio values below 0.9 are nonphysical and thus sorted out. Operating conditions: 150 bar injection with a duration of $2 \mathrm{~ms}$; atmosphere with 10 bar and $400 \mathrm{~K}$.

Recorded images are evaluated with regard to temperature only. The post-processing is simplified compared to the droplet evaluation, because no droplets need to be isolated. Fig. 5(A) shows both single-shot intensity images of the spray and Fig. 5(B) their corresponding ratio. Since rotational symmetry of the spray is assumed, only half the illuminated cut-out section is recorded ${ }^{11}$. Both intensity images are affected by multiply scattered light, detected as "noisy" fluorescence on the inside of the hollow-cone. Multiply scattered light is a common phenomenon of hollowcone sprays and can only be suppressed by applying SLIPI ${ }^{12}$. The ratio of both intensity images is affected by multiply scattered light as well. Division of two "noisy" intensity values results in a ratio of in average one. According to the temperature calibration, a ratio value of one corresponds to a fluid temperature of about $350 \mathrm{~K}$ and thus a valid temperature ${ }^{13}$. Therefore, regions of multiply scattered light cannot be clearly distinguished from the actual spray. In order to minimize influences from multiply scattered light, ratio images are filtered. Pixels with ratio values lower than 0.9 are sorted out. This value has been chosen for two reasons: i) Corresponding temperature values of $365 \mathrm{~K}$ and above are too high to occur in the spray and thus, are nonphysical. This is obvious, when the temperature field of the multiply scattered light region is compared with the temperature values of the actual spray. ii) It is commonly known, how liquid spray formation has to look like in the near nozzle region without multiply scattered light. Using the ratio-threshold of 0.9 allows to cut-out regions where liquid is not supposed to be (compare fig. $5 \mathrm{~A}$ and $\mathrm{B}$ ).

Fig. 6 shows four average temperature-images for differernt moments of the fuel injection. Although images are refined by the ratio-threshold filter, regions of multiply scattered light are still visible as zones of nonphysical high temperatures. Temperature images allow identification of consequences of flow phenomena such as a temperature change due to a toroidal vortex (Fig. 6, C).

In the following, measured temperature fields are judged with regard to credibility. During mixture formation of the spray, droplets are expected to increase their temperature continuously ${ }^{14}$. However, spray images reveal the

\footnotetext{
${ }^{11}$ The injector is therefore located in the top right corner of the images.

${ }^{12}$ Structured Laser Illumination Planar Imaging, see [5] for CW-2D-2cLIF thermometry combined with SLIPI.

${ }^{13} \mathrm{Ambient}$ pressure of 10 bar causes an increased boiling point of Ethanol of $424 \mathrm{~K}$.

${ }^{14}$ The initial droplet temperature is assumed to be below adiabatic droplet temperature.
} 

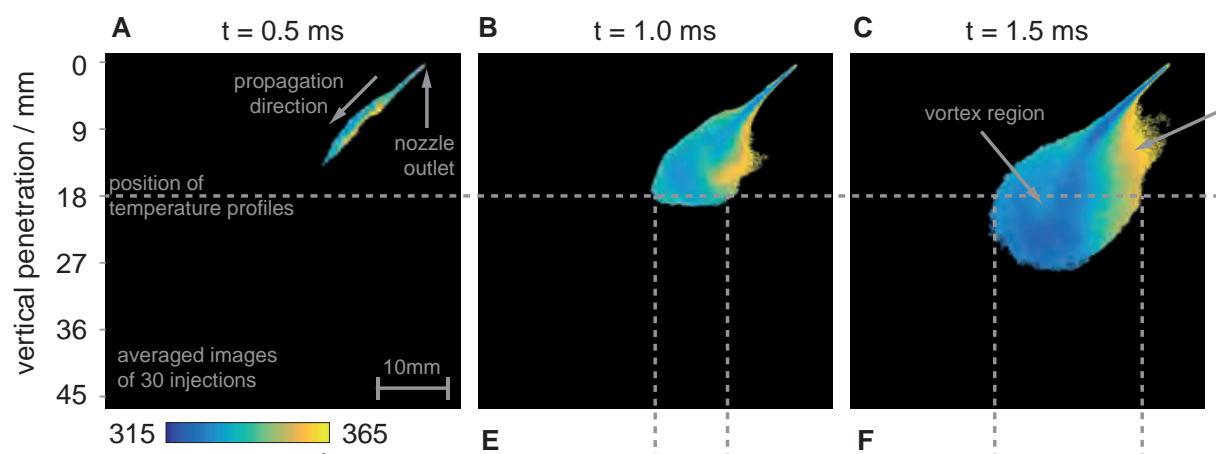

temperature / $\mathrm{K}$
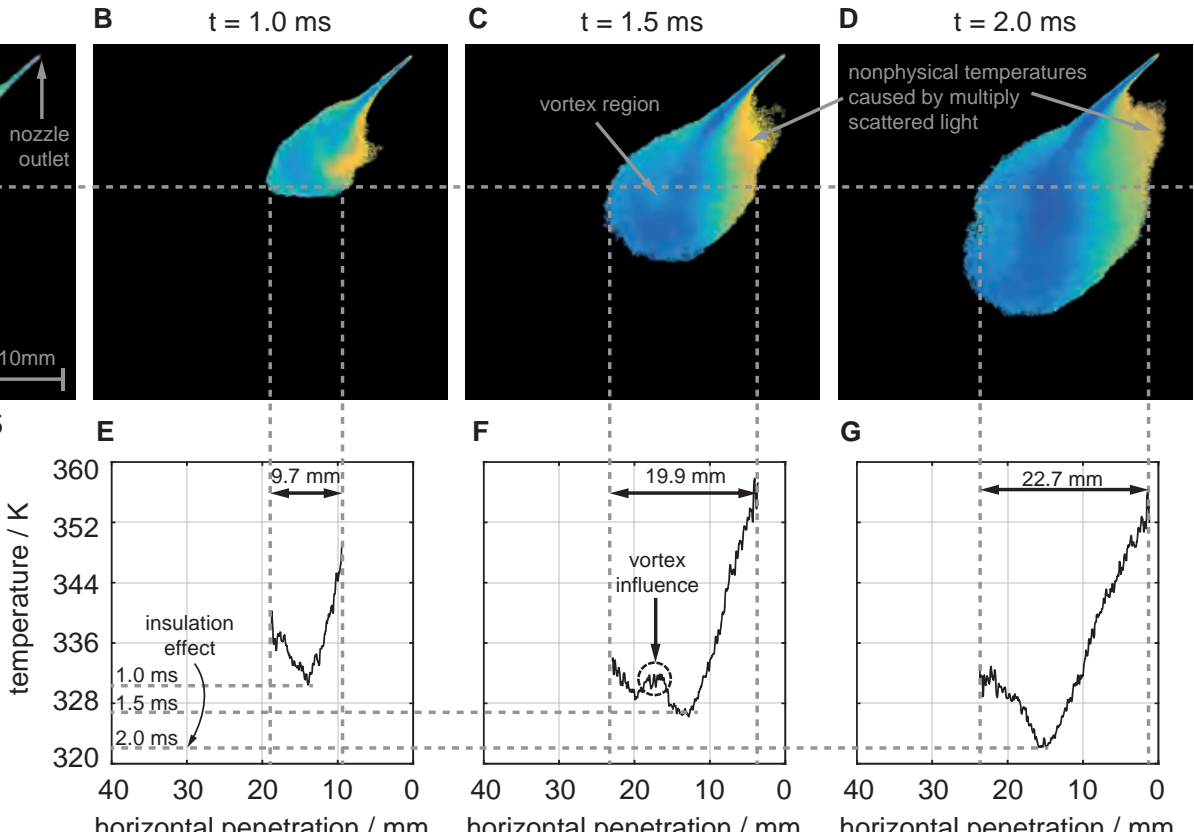

horizontal penetration / $\mathrm{mm}$

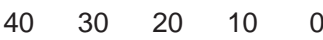

horizontal penetration / $\mathrm{mm}$

horizontal penetration / $\mathrm{mm}$

Figure 6. Average temperature images of the spray propagation in four time steps after start of injector energizing (A-D). All images show signs of multiply scattered light on the cone's inside. Comparison of different time steps needs consideration of the fact that not droplets, but positions are followed over time. As the spray propagates, the spray-sheet-core becomes more and more insulated by liquid and vapor. As a consequence droplet heating is slowed down. Diagrams $(\mathbf{E})$ to $(\mathbf{G})$ quantify the spray's diffusion-limited mixture formation by comparing temperature profiles $18 \mathrm{~mm}$ below injector outlet. Operating conditions: 150 bar injection with a duration of $2 \mathrm{~ms}$; atmosphere with 10 bar and $400 \mathrm{~K}$.

opposite: a more or less isothermal spray-sheet-core that cools down with an advancing time after start of injection (Fig. $6 \mathrm{~A}$ to D). To resolve this contradiction, content of temperature-images must be interpreted: Comparing images of different times steps need consideration of the fact that only fixed positions are compared and not droplets over their lifetime. A thought experiment helps to understand this statement: Only one fixed position below the injector outlet shell be evaluated. Fuel droplets that arrive at this position are pushing away previous droplets, which become an insulating layer of liquid and vapor for the new droplets. Due to the insulation layer, no hot unsaturated air reaches the new droplets. Heating at the fixed position is therefore decelerated. Basically, this progress describes a diffusion-limited mixture formation, where evaporation is much faster than heating and air-vapor mixing is hindered. Occurrence of this mechanism depends on the type of nozzle and injection and ambient conditions.

Diagrams $E$ to $G$ of fig. 6 quantify the insulation-effect for the recorded spray temperature fields of this work. The diagrams show temperature profiles of the spray, evaluated $18 \mathrm{~mm}$ vertically below the injector outlet for three different time steps of the injection. All graphs show the coldest spray region in the center of the spray-sheet. Horizontal positions further distant from the sheet-core have higher temperatures, because the insulation layer is thinner. For a later moment of the injection ( $E$ to $F$ ), the spray-sheet becomes wider. A wider spray-sheet has a thicker insulation layer and thus a lower sheet-core temperature. The entire process is superimposed by horizontal movement of the spray-cloud and occurrence of a toroidal vortex that is positioned at the outer side of the hollowcone. Diagram $6(F)$ indicates the influence of the vortex as local temperature increase. The vortex transports hot unsaturated air inside the spray-sheet-core. A higher heat flux is assumed to increase droplet heating.

In total, temporal changes of the spray temperature seem to be reasonable. The overall temperature level is credible as well: Coldest spray temperatures are always above injection temperature (305 K). Highest spray temperatures (excluding regions of multiply scattered light) are below the boiling point of Ethanol ( $424 \mathrm{~K}$ at 10 bar) and more importantly below adiabatic droplet temperature of the selected ambient condition $(340 \mathrm{~K})^{15}$.

\section{Conclusions}

This work presents the development of a new temperature measurement method named pulsed planar two color laser-induced fluorescence thermometry with MDR-enhanced energy transfer or in short: pulsed 2D-2cLIF-EET. The method is based on a previous 1D-spectroscopic version that is specifically designed for droplet applications, where morphology dependent resonances (MDRs) are suppressed to enable a reasonable temperature measurement. Novelty of this work is the planar detection setup. The second detection dimension extends the number of possible research-applications significantly - fast and turbulent processes, e.g. engine-like sprays, can now be measured at once in size and temperature. The method design of this work is presented step-wise from initial dye selection that is required for MDR-shifting, over explanation to the required equipment, to two application cases that led to new findings and open questions:

\footnotetext{
${ }^{15}$ The value is determined by applying an analytic one dimensional single droplet evaporation model.
} 
- Droplet application: Temperature imaging of micro droplets shows overall reliable results. However, locally resolved average droplet images reveal a systematic temperature fluctuation across isothermal droplets that is nonphysical and thus must be caused by the measurement technique. The cause of this phenomenon is presumably based on local changes of fluorescence excitation intensity. Further research would allow to optimize the droplet application and also answer open research questions of other publications.

- Macroscopic spray application: Spray investigations show reasonable temperature results as well. Temporal temperature changes of the hollow-cone spray reveal a diffusion-limited mixture formation process. Main challenge of the spray application are issues due to multiply scattered light that causes "noisy" fluorescence. Corresponding spray regions on the spray-cone's inside appear as zones with nonphysical high temperatures. Only a SLIPI extension of the setup may solve the problem.

\section{Acknowledgement}

This work was performed as part of the Cluster of Excellence "Tailor-Made Fuels from Biomass", which is funded by the Excellence Initiative of the German federal and state governments to promote science and research at German universities.

\section{References}

[1] Lemoine, F., Antoine, Y., Wolff, M., and Lebouche, M., 1999. "Simultaneous temperature and 2d velocity measurements in a turbulent heated jet using combined laser-induced fluorescence and Ida". Experiments in Fluids, 26(4), pp. 315-323.

[2] Palmer, J., Reddemann, M. A., Kirsch, V., and Kneer, R., 2016. "Temperature measurements of micro-droplets using pulsed 2-color laser-induced fluorescence with mdr-enhanced energy transfer". Experiments in Fluids, $57(12)$.

[3] Chang, R. K., and Campillo, A. J., eds., 1996. Optical Processes in Microcavities. Advanced Series in Applied Physics. WORLD SCIENTIFIC.

[4] Dunand, P., Castanet, G., and Lemoine, F., 2012. "A two-color planar lif technique to map the temperature of droplets impinging onto a heated wall". Experiments in Fluids, 52(4), pp. 843-856.

[5] Mishra, Y. N., Abou Nada, F., Polster, S., Kristensson, E., and Berrocal, E., 2016. "Thermometry in aqueous solutions and sprays using two-color lif and structured illumination". Optics Express, 24(5), p. 4949.

[6] Deprédurand, V., Miron, P., Labergue, A., Wolff, M., Castanet, G., and Lemoine, F., 2008. "A temperaturesensitive tracer suitable for two-colour laser-induced fluorescence thermometry applied to evaporating fuel droplets". Measurement Science and Technology, 19(10), p. 105403.

[7] Palmer, J., Mathieu, F., Reddemann, M., and Kneer, R., 08.- 10. Spetember 2014. "Temperature measurements of evaporating biofuel droplets". ILASS - Europe, 26th Annual Conference on Liquid Atomization and Spray Systems, Bremen - Germany.

[8] Lavieille, P., Delconte, A., Blondel, D., Lebouché, M., and Lemoine, F., 2004. "Non-intrusive temperature measurements using three-color laser-induced fluorescence". Experiments in Fluids, 36(5), pp. 706-716.

[9] Zhang, G., Hung, D. L. S., and Xu, M., 2014. "Experimental study of flash boiling spray vaporization through quantitative vapor concentration and liquid temperature measurements". Experiments in Fluids, 55(8).

[10] Chaze, W., Caballina, O., Castanet, G., and Lemoine, F., 2016. "The saturation of the fluorescence and its consequences for laser-induced fluorescence thermometry in liquid flows". Experiments in Fluids, 57(4).

[11] Tzeng, H.-M., Wall, K. F., Long, M. B., and Chang, R. K., 1984. "Evaporation and condensation rates of liquid droplets deduced from structure resonances in the fluorescence spectra". Optics Letters, 9(7), p. 273.

[12] Wolff, M., Delconte, A., Schmidt, F., Gucher, P., and Lemoine, F., 2007. "High-pressure diesel spray temperature measurements using two-colour laser-induced fluorescence". Measurement Science and Technology, 18(3), pp. 697-706.

[13] Perrin, L., Castanet, G., and Lemoine, F., 2015. "Characterization of the evaporation of interacting droplets using combined optical techniques". Experiments in Fluids, 56(2).

[14] Serpenguzel, A., Kucuksenel, S., and Chang, R., 2002. "Microdroplet identification and size measurement in sprays with lasing images". Optics Express, 10(20), p. 1118.

[15] Anand, M., Dharmadhikari, A., Dharmadhikari, J., Mishra, A., Mathur, D., and Krishnamurthy, M., 2003. "Twophoton pumped lasing from methanol micro-droplets doped by a weakly fluorescent dye". Chemical Physics Letters, 372(1-2), pp. 263-268.

[16] Chen, G., Mohiuddin Mazumder, M., Chang, R. K., Christian Swindal, J., and Acker, W. P., 1996. "Laser diagnostics for droplet characterization: Application of morphology dependent resonances". Progress in Energy and Combustion Science, 22(2), pp. 163-188.

[17] Tzeng, H.-M., Wall, K. F., Long, M. B., and Chang, R. K., 1984. "Laser emission from individual droplets at wavelengths corresponding to morphology-dependent resonances". Optics Letters, 9(11), p. 499.

[18] Labergue, A., Pena-Carillo, J.-D., Gradeck, M., and Lemoine, F., 2017. "Combined three-color lif-pda measurements and infrared thermography applied to the study of the spray impingement on a heated surface above the leidenfrost regime". International Journal of Heat and Mass Transfer, 104, pp. 1008-1021.

[19] Frackowiak, B., and Tropea, C., 2010. "Numerical analysis of diameter influence on droplet fluorescence". Applied optics, 49(12), pp. 2363-2370. 